\title{
Relationship between Dysphagia and Serum Substance P Level in Chronic Central Nervous Disease
}

\author{
Yoshiyuki Kishida, Naoto Maeda, Yoshikazu Murawaki
}

Second Department of Internal Medicine, Tottori University School of Medicine, Yonago, Japan.

Email: sasaki2009@bloom.ocn.ne.jp

Received December $17^{\text {th }}, 2012$; revised January $18^{\text {th }}, 2013$; accepted January $25^{\text {th }}, 2013$

\begin{abstract}
Purpose: We compared serum substance P (SP) levels in underlying diseases and dysphagia, or its absence, in patients with cerebrovascular disease, neurodegenerative disease or Alzheimer's disease, to investigate the relationship between dysphagia and serum SP in chronic central nervous disease. Methods: Subjects comprised 94 patients admitted to a hospital or nursing home during the 5 years between April 2007 and April 2012 with central nervous symptoms. Serum SP levels were measured by enzyme immunoassay, and video endoscopy using a nasal endoscope in all subjects to objectively evaluate swallowing function. Results: Serum SP level was very similar in central nervous disease without dysphagia and controls without central nervous disease. Conversely, serum SP level was significantly lower in central nervous disease with dysphagia. When comparing underlying diseases, serum SP was significantly lower in Parkinson's disease than in other disease groups (cerebrovascular disease, Alzheimer's disease). Looking at changes in serum SP levels over time after disease onset, SP level was significantly low in subjects without dysphagia at the time of onset who went on to develop dysphagia during the disease course, whereas serum SP level tended to be higher in subjects with dysphagia at the time of onset and improvement during the disease course. With Parkinson's disease and cerebrovascular disease, serum SP was low, particularly in subjects thought to have severe damage to the basal ganglia. Conclusion: Serum SP is generally thought to decrease in patients with cerebrovascular disease accompanied by dysphagia, but these results suggest that serum SP levels can be expected to improve to some extent, even if dysphagia is present at disease onset, assuming, for example, that some basal ganglia function remains. Positive therapeutic interventions such as swallowing rehabilitation should be promoted in such patients, with the goal of improving swallowing function.
\end{abstract}

Keywords: Substance P; Dysphagia; Central Nervous Disease

\section{Introduction}

Together with motor function and higher brain function, swallowing is one of the basic human functions, but could be considered the most important in terms of a direct connection to survival. The rapid increase in the elderly population in recent years has been accompanied by a large number of cases involving cerebrovascular diseases, neuromuscular diseases such as Parkinson's disease, and age-related brain changes. In many cases, swallowing function is impaired as a result. Such impairments increase the risk of aspiration pneumonia and choking $[1,2]$ during oral intake of nutrients. In Japan, nasogastric intubation or gastrostomy is therefore standard practice to manage dysphagia arising from pathological changes in the higher brain. Because some patients develop serious complications such as aspiration pneumonia through reflux of enteral nutrients, further study of the suitability of gastrostomy as a means of ad- ministering nutrition in dysphagic patients is needed. Ideally such patients would regain the ability to take nutrients orally by recovering from dysphagia, but no obvious, measurable markers have been identified to indicate those patients most likely to recover.

Substance P (SP) is a neurotransmitter belonging to the tachykinin peptide family. Widely distributed throughout the peripheral and central nervous systems of vertebrates, $\mathrm{SP}$ is known to have a wide range of biological activities: as well as contributing to pain transmission via primary afferent neurons in the peripheral nerves, and to emotional responses such as depression, anxiety and fear by bonding with neurokinin 1 (NK1) receptors in the central nervous system, it also acts directly on immune cells to modify inflammatory and immune responses.

Recent findings have also shown that SP may be a biological indicator of dysphagia [3,4], based on the fact that SP is present in vagal non-cholinergic nerve fibers 
innervating the respiratory tract [5] and is involved in mechanisms for preventing aspiration, such as the cough reflex and swallowing movements. In fact, reports have described decreased serum ST levels in patients with cerebrovascular disease involving dysphagia [6,7]. The present investigation examined the relationship between serum SP levels and presence or absence of dysphagia, and discusses the significance of serum SP levels in chronic dysphagia.

\section{Subjects and Methods}

\subsection{Subjects}

Subjects comprised 94 patients in admitted to a hospital or nursing home during the 5 years between April 2007 and April 2012 with central nervous symptoms due to conditions including cerebrovascular disease, Parkinson disease and Alzheimer's disease, along with a control group of 4 subjects without central nervous symptoms. When, in addition to the underlying disease, all symptoms and findings were stable, we examined whether the subject had dysphagia, and measured serum SP levels at the same time. For 17 subjects $(18.0 \%)$, we also performed the same examinations and measurements 3 years after first measurement.

Each subject or their family received a written explanation of the research and provided written consent before enrolment in the study.

\subsection{Evaluation of Swallowing Function}

We performed video endoscopy (VE) using a nasal endoscope (Olympus Medical Systems ENT-GP, Tokyo, Japan) in all subjects to objectively evaluate swallowing function. After nasal insertion of the endoscope, the in-

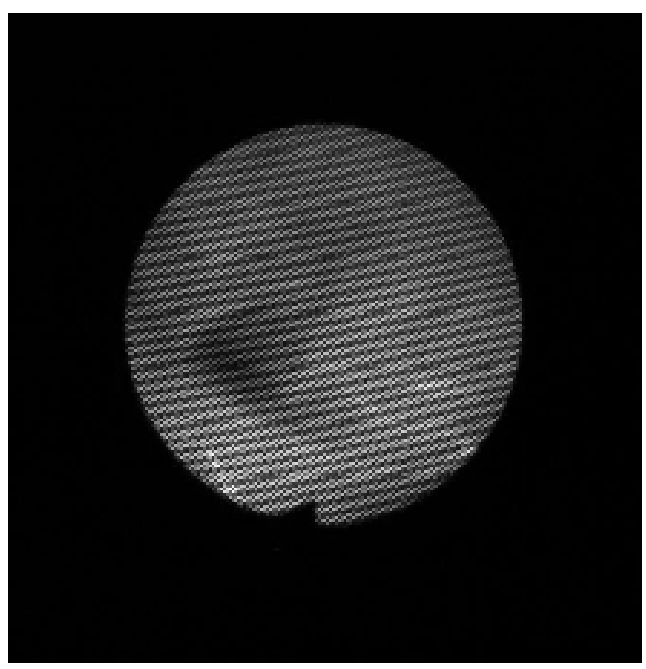

(a) ternal structures were examined for organic abnormalities of the pharynx and larynx, operation of the airway defense mechanisms, movement ability of organs of swallowing and presence or absence of aspiration, and these were evaluated in accordance with the evaluation form proposed by the Japanese Society of Dysphagia Rehabilitation (http://info.fujita-hu.ac.jp/\%7Erehabmed/jsdr/) (Figure 1).

\subsection{Measurement of Serum SP Levels}

SP serum levels were measured by enzyme immunoassay (Substance P Enzyme Immunoassay Kit; Assay Designs, MI, USA) using blood samples taken in the morning before breakfast during regular health monitoring at least 6 months after symptoms had reached a stage where there was considered to be no possibility of improvement.

\subsection{Statistical Methods}

Prism 5 for Mac OS X version 5 software (Tokyo, Japan) was used for statistical analyses. Student's t-test was used for intergroup comparisons based on sex differences, and Pearson's correlation coefficient was used to evaluate associations between age and serum SP level, and between serum albumin and serum SP levels. The difference of SP and albumin among diseases was tested by one-way analysis of variance. A significance level of $\mathrm{P}<$ 0.05 was set for each test.

\section{Results}

\subsection{Subject Background Factors (Table 1)}

Table 1 shows the background and clinical data of the subjects. The 94 subjects (17 men, 77 women) showed a

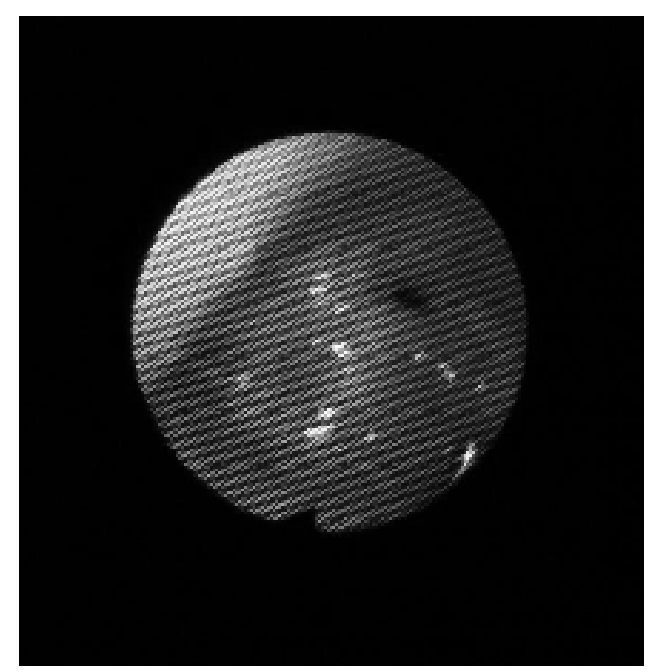

(b)

Figure 1. Findings of the endoscopic evaluation of swallowing function. (a) Inflow of the saliva into the glottis. (b) Backflow of the liquid content from the tracha. 
Table 1. Background and clinical data of patients.

\begin{tabular}{|c|c|c|c|c|c|}
\hline & $\begin{array}{c}\mathrm{N} \\
(\mathrm{M}: \mathrm{F})\end{array}$ & Age & $\mathrm{SP}(\mathrm{pg} / \mathrm{ml})$ & Albumin $(\mathrm{g} / \mathrm{dl})$ & Dysphagia (\%) \\
\hline $\begin{array}{c}\text { All } \\
\text { cases }\end{array}$ & $\begin{array}{c}94 \\
(17: 77)\end{array}$ & $84.6 \pm 8.6$ & $114.4 \pm 50.4$ & $3.8 \pm 0.5$ & $38(40.4)$ \\
\hline CVD & $\begin{array}{c}31 \\
(6: 25)\end{array}$ & $83.0 \pm 7.5$ & $130.1 \pm 56.6$ & $3.7 \pm 0.6$ & $12(38.7)$ \\
\hline $\mathrm{AD}$ & $\begin{array}{c}50 \\
(8: 42)\end{array}$ & $86.9 \pm 7.4$ & $108.0 \pm 44.4$ & $3.8 \pm 0.5$ & $17(34.0)$ \\
\hline PD & $9(1: 8)$ & $79.4 \pm 14.8$ & $868.0 \pm 14.3$ & $3.5 \pm 0.5$ & $9(100)$ \\
\hline Control & $14(2: 2)$ & $81.1 \pm 5.5$ & $164.8 \pm 40.5$ & $4.3 \pm 0.4$ & $0(0)$ \\
\hline
\end{tabular}

SP: serum substance $\mathrm{P}$ concentration (mean $\pm \mathrm{SD}$ ); $\mathrm{CVD}$ : cerebral vascular disease; AD: Alzheimer's disease; PD: Parkinson's disease.

mean age of $84.6 \pm 8.6$ years, serum SP $114.4 \pm 50.4$ $\mathrm{pg} / \mathrm{mL}$, and albumin $3.8 \pm 0.5 \mathrm{~g} / \mathrm{dL}$. Dysphagia was confirmed in 38 subjects (40.4\%). Dividing these subjects into four groups by disease (cerebrovascular disease, Alzheimer's disease, Parkinson's disease, or control group), there were 31 subjects ( 6 men, 25 women) in the cerebrovascular disease group, 50 subjects $(8$ men, 42 women) in the Alzheimer's disease group, 9 subjects (1 man, 8 women) in the Parkinson's disease group and 4 control group subjects (2 men, 2 women). Serum SP levels in these groups were $130.1 \pm 56.6 \mathrm{pg} / \mathrm{mL}$ for cerebrovascular disease, $108.0 \pm 44.4 \mathrm{pg} / \mathrm{mL}$ for Alzheimer's disease, $68.0 \pm 14.3 \mathrm{pg} / \mathrm{mL}$ for Parkinson's disease and $164.8 \pm 40.5 \mathrm{pg} / \mathrm{mL}$ for healtly elders, respectively, with significantly lower levels for the Parkinson's disease group $(\mathrm{P}<0.05)$ than for the cerebrovascular disease or Alzheimer's disease groups. No significant differences in serum SP levels were identified between the cerebrovascular disease, Alzheimer's disease and control groups.

\subsection{Sex differences in SP Levels}

No obvious sex differences in serum SP levels were evident, with mean levels of $108.4 \pm 47.7 \mathrm{pg} / \mathrm{mL}$ for men and $115.7 \pm 51.2 \mathrm{pg} / \mathrm{mL}$ for women.

\subsection{SP levels and Age}

No obvious correlations were seen between age and serum $\mathrm{SP}$ level $(\mathrm{r}=-0.08, \mathrm{P}=0.44)$.

\subsection{Association between Dysphagia and Serum SP}

When comparing serum SP levels in subjects with and without dysphagia, levels were significantly lower in the 38 subjects with dysphagia $(78.9 \pm 34.5 \mathrm{pg} / \mathrm{mL})$ than in the 56 subjects with central nervous disorders but no dysphagia $(138.4 \pm 44.9 \mathrm{pg} / \mathrm{mL} ; \mathrm{P}<0.001)$.

\subsection{Presence/Absence of Dysphagia and Changes in Serum SP Level over Time}

To examine changes in serum SP level over the course of dysphagia, we investigated serum SP levels 3 years after initial measurement in 10 subjects without dysphagia at the onset of underlying disease and in 7 subjects with dysphagia at disease onset.

Five of the 10 subjects without dysphagia at disease onset developed dysphagia during the course of the disease. In this group (cerebrovascular disease, $\mathrm{n}=1$; Alzheimer's disease, $\mathrm{n}=4$ ), serum SP levels fell significantly from $119 \pm 15.7 \mathrm{pg} / \mathrm{mL}$ to $66.7 \pm 15.1 \mathrm{pg} / \mathrm{mL}(\mathrm{P}<$ 0.001; Figure 2). In contrast, among the 7 subjects with dysphagia at disease onset, three (all cerebrovascular disease patients) showed improvement in dysphagia during the disease course, and serum SP levels in these 3 subjects followed an upward trend, rising from $102.8 \pm$ $24.6 \mathrm{pg} / \mathrm{mL}$ to $173 \pm 56.9 \mathrm{pg} / \mathrm{mL}(\mathrm{P}=0.053$; Figure 3$)$. The remaining 4 subjects showed no improvement in dysphagia, and all had severe damage to the basal ganglia.

\section{Discussion}

This study found no differences in serum SP levels between subjects with chronic central nervous disease but not dysphagia and a control group with no central nervous disease. However, when comparing diseases, subjects with the degenerative Parkinson's disease showed serum SP levels significantly lower than those with cerebrovascular disease. This may be partly due to the fact that Parkinson's disease, which involves damage to the basal ganglia, is accompanied by decreases in dopamine, which is released from the substantia nigra, and the neurotransmitter SP [4]. In fact, this applies not only to Parkinson's disease, but also to patients with significant damage to the basal ganglia due to cerebrovascular disease [3]. Such patients are thought to be susceptible to

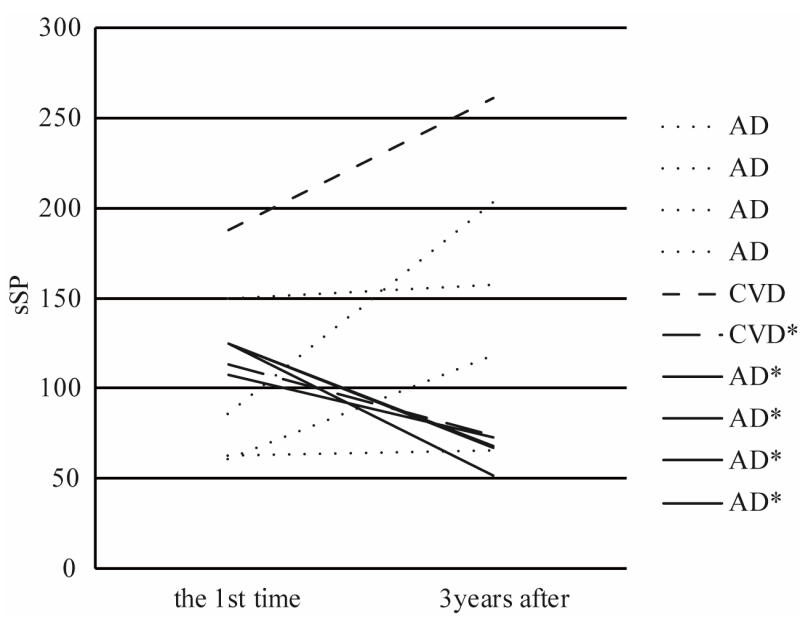

Figure 2. Temporal change of the serum SP value of cases without dysphagia. ${ }^{*}$ The case developed dysphagia during the course of the disease. Ssp: serum sunbstance $P$ concentration (mean $\pm \mathrm{SD}$ ); CVD: cerebral vasoular disease; $\mathrm{AD}$ : Alzheimer's disease; PD: Parkinson's disease. 


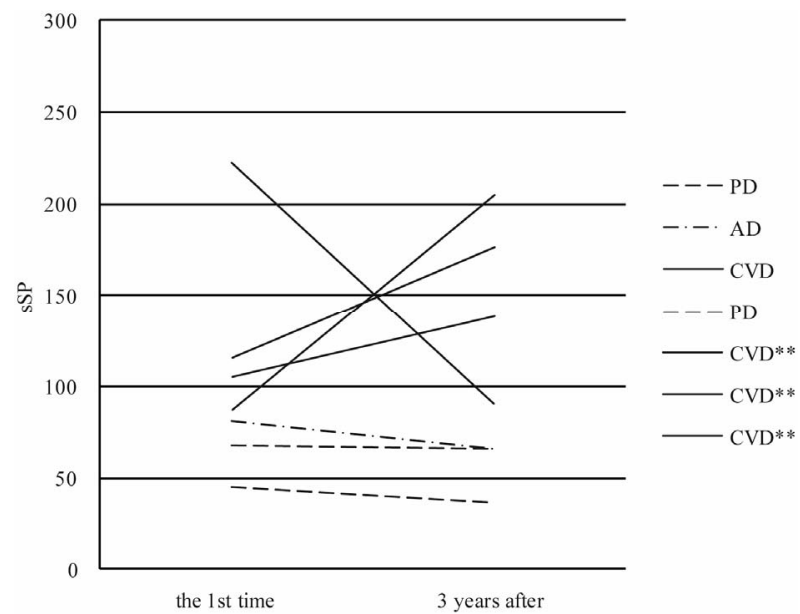

Figure 3. Temporal change of the serum SP value of cases with dysphagia. ${ }^{* *}$ The case showed improvement in dysphagia during the disease course. sSP: serum sunbstance $P$ concentration (mean \pm SD); CVD: cerebral vasoular disease; AD: Alzheimer's disease; PD: Parkinson's disease.

aspiration because the swallowing reflex and cough reflex are unable to function normally due to the low levels of SP in the blood. This represents a major cause of agerelated pneumonia [1]. Dysphagia has been treated with ACE (Angiotensin converting enzyme) inhibitors, which increase SP levels in peripheral nerves by blocking SPdegrading enzymes, and by pharyngeal administration of a substance such as capsaicin (the active component of chili peppers), which increases SP levels in the oral cavity and respiratory tract via afferent $\mathrm{C}$ fibers, thereby promoting the swallowing reflex $[8,9]$. However, these treatments are only thought to be effective in patients assumed to have low SP production due to "partial" damage to the basal ganglia.

When looking at changes over the clinical course of dysphagia, serum SP levels were significantly low in subjects without dysphagia at time of onset who went on to develop dysphagia during the course of the disease, whereas serum SP showed an upward trend in subjects with dysphagia that was present at time of onset but showed improvement during the course of the disease. This indicates a negative correlation between dysphagia and serum SP level. Furthermore, all subjects in whom dysphagia resolved showed cerebrovascular disease, whereas all Parkinson's disease patients failed to show any improvement in dysphagia and also exhibited virtually unchanged serum SP levels. This may be due to differences in the extent of functional loss in the basal ganglia in Alzheimer's disease, Parkinson's disease and cerebrovascular disease. In addition, patients with Parkinson's disease would be expected to show a persistent, progressive decline in SP, since Parkinson's is a progressive degenerative disease.

These findings suggest that among patients with chro- nic central nervous disorders involving dysphagia, those with complete loss of basal ganglia function will have persistently low serum SP levels. On the other hand, patients with an intact basal ganglia are likely to sustain the capacity for SP production, who have the possibility that serum SP levels wound rise with recovery from dysphagia. This means that when treating patients with an intact basal ganglia, medical interventions such as gastrostomy and swallowing rehabilitation should be actively considered in the expectation that symptoms will resolve through physicochemical stimulation of the intestinal tract. However, it has been pointed out that a gastrostomy installed in the early stage of cerebrovascular disease can lead to a loss of swallowing function, and also results in an increased risk of aspiration pneumonia as a result of gastroesophageal reflux following functional declines in gastric secretion $[10,11]$. This problem could be overcome by providing parenteral nutrition for dysphagia when it occurs at the onset of cerebrovascular disease, then introducing a gastrostomy after the disease has stabilized.

A number of limitations must be considered when interpreting the present results: the number of subjects was small; the causal relationships with the underlying diseases were unclear; the study design was not prospective; and the effects of age may have introduced a type II error (false-negative error). Age and sex differences in serum SP levels have not been indicated in previous studies, and we similarly dispensed with discussion of the effects of sex and age on serum SP levels. However, almost no research has been reported on swallowing function in the chronic phase. Therefore, in the investigation of the link between serum SP levels and improvement of dysphagia, this study is of considerable importance as preliminary research in this area.

\section{Conclusion}

We demonstrated a strong link between dysphagia and serum SP levels. In cerebrovascular disease involving dysphagia, complete loss of basal ganglia function would be unexpected, at least in patients with normal serum SP levels at disease onset. This suggests that medical interventions such as gastrostomy and swallowing rehabilitation should be actively considered on the expectation that dysphagia symptoms will resolve through physicochemical stimulation of the intestinal tract. This subject demands further research, including a prospective study.

\section{REFERENCES}

[1] S. Teramoto, Y. Fukuchi, H. Sasaki, K. Sato, K. Sekizawa and T. Matsuse, "High Incidence of Aspiration Pneumonia in Community- and Hospital-Acquired Pneumonia in Hospitalized Patients: A Multicenter, Prospec- 
tive Study in Japan," Journal of the American Geriatrics Society, Vol. 56, No. 3, 2008, pp. 577-579. doi:10.1111/j.1532-5415.2008.01597.x

[2] M. Yamawaki, "Prevalence of Aspiration Pneumonia," Sogo Riha, Vol. 37, 2009, pp. 105-109.

[3] T. Nakagawa, T. Ohrui, K. Sekizawa and H. Sasaki, "Sputum Substance P in Aspiration Pneumonia," Lancet, Vol. 345, No. 8962, 1995, p. 1447. doi:10.1016/S0140-6736(95)92638-0

[4] T. Nakagawa, K. Sekizawa, H. Arai, R. Kikuchi, K. Manabe and H. Sasaki, "High Incidence of Pneumonia in Elderly Patients with Basal Ganglia Infarction," Arch International Medicine, Vol. 157, No. 3, 1997, pp. 321-324. doi:10.1001/archinte.1997.00440240085013

[5] Y. S. Lee, K. M. Km, C. Shin, J. J. Shim, K. H. Kim, H. K. Kang, H. S. Yoo and H. K. In, "Substance P-Immunoreactive Nerves in Endobronchial Biopsies in CoughVariant Asthma and Classic Asthma," Respiration, Vol. 70, No. 1, 2003, pp. 49-53. doi:10.1159/000068413
[6] T. Nagamine, "Serum Substance P Levels in Patients with Chronic Schizophrenia Treated with Typical or Atypical Antipsychotics," Neuropsychiatric Disease and Treatment, Vol. 4, No. 1, 2008, pp. 289-294. doi:10.2147/NDT.S2367

[7] S. Matsumoto, S. Shimodo and K. Kawahira, "Substance P," Geriatric Medicine, Vol. 45, 2007, pp. 1331-1335.

[8] Y. Jin, K. Sekizawa, T. Fukushima, M. Morikawa, H. Nakazawa and H. Sasaki, "Capsaicin Desensitization Imhibits Swallowing Reflex in Guinea Pigs," American Journal of Respiratory and Critical Care Medicine, Vol. 149, No. 1, 1994, pp. 261-263.

[9] D. Ramsey, D. Smithard and L. Kalra, "Silent Aspiration: What Do We Know?" Dysphgia, Vol. 20, No. 3, 2005, pp. 218-225. doi:10.1007/s00455-005-0018-9

[10] T. Kisa, Rinsyoueiyou, Vol. 106, 2005, pp. 327-333.

[11] S. Teramoto, "Aspiration Pneumonia May Not Be Prevented by Gastrostmyalone," Journal of Clinical Rehabilitation, Vol. 17, 2008, pp. 826-829. 